\title{
A STANKO SKKERLJ POUR SES QUATRE-VINGTS ANS
}

Né à Novo mesto en Slovénie, le 7 février 1893, Stanko Škerlj, après avoir terminé les études élémentaires et secondaires dans sa ville natale, commence, à Vienne, des études des langues et littératures germaniques et romanes. Bientôt, la romanistique finit par occuper la place la plus importante; aussi, l'italien devient-il la langue de ses études, celle de sa vie.

Sa formation scientifique date d'une époque dans laquelle une scission entre la linguistique et l'histoire littéraire n'était pas encore absolument obligatoire et de tant moins la diramation entre la philologie et la linguistique. Sa thèse de doctorat «Rapports entre la plus ancienne lyrique italienne et le moyen-haut-allemand Minnesang» est une recherche littéraire. Dans une certaine mesure, il reste fidèle à la littérature; en témoignent ses études sur Dante, Pétrarque, Léonarde, Machiavelli, Cellini et Goldoni. En témoigne, en outre, un constant intérêt pour les relations entre la culture italienne et celles des pays slaves du Sud, et plus particulièrement la slovène. Le fruit le plus mûr de cette direction d'études - la première publication dans ce genre de l'histoire date du 1934 - est «Le théâtre italien à Ljubljana dans les siècles passés», un aperçu de l'histoire culturelle de notre peuple, et non seulement de celle du théâtre.

Pourtant, tout Škerlj se trouve dans les oeuvres linguistiques. Si, de premier abord, il partage son -intérêt, désormais consacré à l'italien, à l'histoire de la littérature et à la linguistique, c'est cette dernière qui commence à prédominer, surtout quant à la syntaxe. Serait-ce une prédisposition naturelle ou bien le fait de rencontrer - jeune professeur au lycée - des questions syntaxiques, dans les textes de classe, qu'il fallait expliquer moyenant l'analyse des structures.

Il avait commencé ses études à Vienne, à l'epoque néogrammairienne, les poursuivit à Florence et, plus tard, à Paris. Des idées nouvelles pullulaient un peu partout, mais encore lentement. Quoiqu'il eût des maîtres insignes (Meyer-Lüble, Ettmayer, E. G. Parodi, F. Brunot, Mario Roques, J. Marouzeau), Škerlj n'a jamais eu des «mentors». Cela eut diverses conséquences: de mauvaises - marche plus lente des investigations, avec des détours, formation plus lente d'un système à lui; mais aussi de bonnes: sûreté des faits établis, clarté au sujet des difficultés de 
l'exploration. Autre conséquence: Škerlj n’a jamais appartenu à une «école» linguistique; il ne se sentait ni lige à une doctrine, ni fort de son appui.

De ses liaisons avec l'orientation néogrammairienne, il s'est libéré facilement, presqu'imperceptiblement. Sa nouvelle voie, d'un côté, paitait de la conviction que la fonction du langage humain est d'exprimer, de manifester et de rendre possible la communication entre les hommes; de l'autre, elle avait pour but de mener à la connaissance la plus profonde possible du fonctionnement réel du langage. L'une des tâches principales - si ce n'est pas simplement la tâche principale - de la linguistique est donc d'étudier par quels moyens linguistiques et dans quelle mesure une langue accomplit sa fonction (qui est surtout de rendre possible la communication). En parenthèse: le langage n'est pas un organisme vivant - comme il est appelé quelquefois -, mais un moyen, un instrument, un outil. Il se développe en même temps que celui qui l'emploie. Cela ne signifie pas que le langage n'ait pas de nécessités propres, des lois particulières, d'autonomie. Cette étude ne se peut pas faire sans confronter continuellement la structure des tournures linguistiques avec ce qu'elles sont obligées à exprimer. Škerlj n'a pas d'allergie à la psychologie en linguistique, pas même à l'introspection. On peut rester sceptique devant beaucoup de «faits» intérieurs qui ne sont «connus» que par l'introspection; mais comment renoncer, en sémantique par ex., à la comparaison de notions sans supposer - disons - un certain parallélisme des aperceptions dans les individus? Elevé dans l'athmosphère néogrammairienne, Škerlj ne peut imaginer une recherche linguistique limitée à un seul état de l'histoire de la langue, serait-ce même si important, comme l'époque contemporaine. Il faut voir un problème dans le temps, car on ne pourrait voir dans un fait linguistique une simple donnée synchronique. Il serait, pourtant, une aveugle croyance dans la diachronie que de renvoyer un problème de langue de quelques siècles en le croyant résolu. La vraie solution, la véritable compréhension des faits de langue repose sur la connaissance de notre vie psychique: ce sont les procès mentaux qui justifient toute innovation.

En introduisant dans la syntaxe le concept de la stéréotypisation, Šlkerlj a été particulièrement heureux avec l'application de ses vues théoriques. Phénomène analogue à la métaphore en sémantique - substitution d'un mot à un autre par similitude du sens - la stéréotypisation est le phénomène syntaxique qui démontre la pauvreté de la langue par rapport à la richesse de la vie psychique et, en même temps, la puissance créatrice de l'homme; exactement comme la métaphore avec laquelle la stéréotypisation a un autre trait commun, celui de l'effet. Enrichir des possibilités expressives du langage, sans élargissement de l'inventaire, employer, pour exprimer deux idées assez éloignées, mais qui ont un trait en commun, la même formule syntaxique sans y rien changer. 
Il y est visible que l'effort constant du linguiste est de parvenir, à travers les moyens de la communication, à la connaissance profonde, complète, autant que possible, du substrat psychique que le parlant a voulu manifester. Dans ce champs, d'après Škerlj, outre l'importance théorique d'une telle direction de la recherche, la linguistique se montre en même temps d'une grande utilité pratique, une matière modulative, qui sert à former l'adolescent. Dans l'apprentissage d'une langue étrangère et, beacoup plus important, de la langue maternelle, une analyse soigneuse de la phrase montre que les moyens d'expression sont plus limités et pauvres qu'exigerait la richesse de notre vie psychique. Celui qui parle se rendra compte qu'il faut peser chaque énonciation avant qu'elle devienne une communication prononcée ou écrite: il cherchera à s'exprimer avec plus de précision et il y prendra l'habitude. Ainsi, la richesse de sa vie interne sera-t-elle presentée à son interlocuteur avec plus d'exactitude.

Les fruits de 60 ans de travail (la première publication remontant à 1915) sont aussi nombreux hors de la discipline de sa vocation. En outre, Stanko Škerlj n'a jamais été détaché des problèmes qui tourmentaient son peuple: une série d'articles sur le caractère ethnique de Trieste, sur les questions de la Carynthie slovène, sur la situation de l'intelligentsia et études universitaires dans un petit peuple démontrent son attachement. au sol slovène.

Les ans paraissent ne pas avoir le poids que leur nombre ferait croire: aussi nous lui souhaitons encore beaucoup d'années fructueuses et espérons de lui tant de travaux précieux pour la linguistique tant romane que générale.

Ses amis 